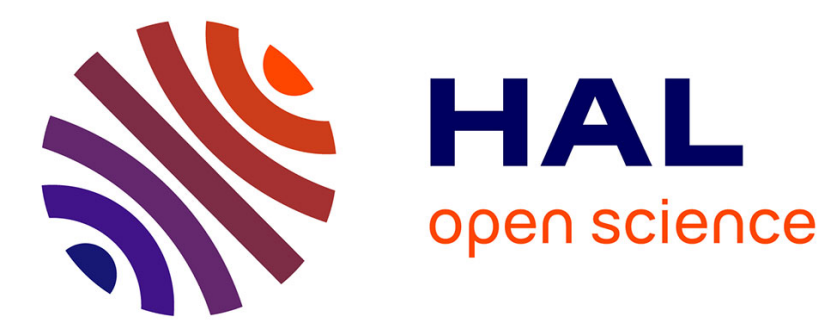

\title{
Ultrafast laser stabilization by nonlinear absorption for enhanced-precision material processing
} Pol Sopeña, Mario Garcia-Lechuga, Andong Wang, David Grojo

\section{To cite this version:}

Pol Sopeña, Mario Garcia-Lechuga, Andong Wang, David Grojo. Ultrafast laser stabilization by nonlinear absorption for enhanced-precision material processing. Optics Letters, 2022, 47 (4), pp.993996. 10.1364/OL.449720 . hal-03576611

\section{HAL Id: hal-03576611 \\ https://hal.science/hal-03576611}

Submitted on 16 Feb 2022

HAL is a multi-disciplinary open access archive for the deposit and dissemination of scientific research documents, whether they are published or not. The documents may come from teaching and research institutions in France or abroad, or from public or private research centers.
L'archive ouverte pluridisciplinaire HAL, est destinée au dépôt et à la diffusion de documents scientifiques de niveau recherche, publiés ou non, émanant des établissements d'enseignement et de recherche français ou étrangers, des laboratoires publics ou privés. 
To be published in Optics Letters:

Title: $\quad$ Ultrafast laser stabilization by nonlinear absorption for enhanced-precision material processing

Authors: Pol Sopeña,Mario Garcia Lechuga,Andong Wang,David Grojo

Accepted: 14 January 22

Posted 14 January 22

DOI: $\quad$ https://doi.org/10.1364/OL.449720

(C) 2022 Optica

To cite this article:

Pol Sopeña, Mario Garcia-Lechuga, Andong Wang, and David Grojo,

"Ultrafast laser stabilization by nonlinear absorption for enhanced-precision material processing," Opt. Lett. 47, 993-996 (2022) 


\title{
Ultrafast laser stabilization by nonlinear absorption for enhanced-precision material processing
}

\author{
Pol Sopeña, ${ }^{1 *}$ Mario Garcia-Lechuga, ${ }^{2,3}$ Andong WANG, ${ }^{1}$ And DaVid \\ GROJO1,4
}

\author{
${ }^{1}$ Aix-Marseille Université, CNRS, LP3, UMR7341, 13009 Marseille, France \\ ${ }^{2}$ Departamento de Física Aplicada, Universidad Autónoma de Madrid, 28049 Madrid, Spain \\ ${ }^{3}$ Centro de Microanálisis de Materiales, Universidad Autónoma de Madrid, 28049 Madrid, Spain \\ ${ }^{4}$ e-mail: david.grojo@univ-amu.fr \\ *Corresponding author: pol.sopena-martinez@univ-amu.fr
}

Received XX Month XXXX; revised XX Month, XXXX; accepted XX Month XXXX; posted XX Month XXXX (Doc. ID XXXXX); published XX Month XXXX

Using ultrafast lasers, sub-diffraction features can be produced thanks to the threshold-based response of materials to the local beam fluence. In practice, Gaussian beams with peak fluence near the modification threshold lead to highresolution. However, this conflicts with reliability as the process becomes increasingly sensitive to pulse-to-pulse energy fluctuations. Using nonlinear absorption in a $\mathrm{ZnS}$ crystal, we demonstrate a passive extra-cavity energy stabilization method in a femtosecond laser material machining configuration. Processing precision and repeatability are enhanced as evidenced by highly reliable amorphous features produced on silicon with sizes ten times smaller than the spot size, becoming a practical solution for high-precision manufacturing applications.

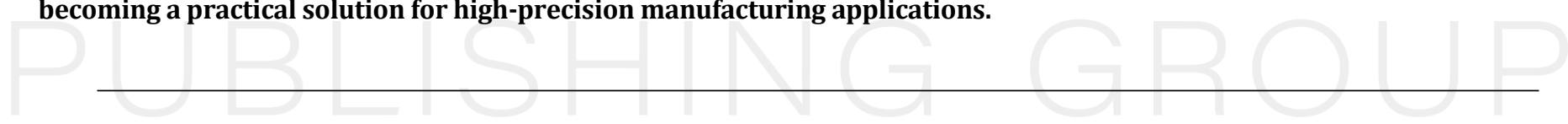

Ultrafast laser micro-processing exploits the ability to locally modify, either structurally or chemically, the surface or bulk of materials by irradiation with focused laser pulses of extremely short durations down to a few femtoseconds [1,2]. These provide high precision as they allow strong-field nonlinear excitation of any solid with energy deposition strictly decoupled to material thermal response, drastically reducing the affected zones and allowing extremely reproducible features. The process switches from a stochastic response driven by non-uniformities with long pulses to a rigorously robust deterministic outcome with femtosecond pulses [3]. All of this is key to achieve sub-diffraction limit resolution in material processing applications, as shown by impressive studies proving resolutions as small as a few tens of nanometers [4,5]. Hereof, ultrafast lasers allow direct fabricating complex structures based on subtractive, additive, or modification processes allowing to address many applications from medicine to electronics [6].

Supported by dielectric surface ablation experiments, we recently confirmed an already well-established threshold-based response when working with ultrashort pulses of <200 fs [7]. An important consequence was an observable incompatible with the concept of nonlinear resolution as confirmed by produced ablation features systematically corresponding to the beam contours at a given threshold intensity independently to the absorption physics [8]. However, these conclusions are not strictly limited to ablation of dielectrics and must hold to all threshold-based modification responses as for instance amorphization. This is directly supported by experimental studies using phase change materials for precise mapping of local fields [9]. Another important conclusion derived from the threshold response in the case of peak-intensity beams, like Gaussian ones, is the possibility of achieving high-resolution features by working at energies near threshold modification fluence $[4,5]$. However, extreme resolution contradicts in practice with reproducibility since the process becomes increasingly dependent on energy fluctuations as we move to peak intensities close to threshold. By applying the error propagation theory on the thresholding problem for a Gaussian incoming profile, we can quantify this aspect and finally express how resolution and reproducibility performances depend on laser energy stability [8].

With these simple considerations, the pulse-to-pulse energy stability of the laser becomes the most critical aspect to improve processing performances. To reduce laser fluctuations, several methods have been reported in various contexts. For example, there are approaches based on the dynamic control of a Pockels cell [10] or Kerr interactions on a crystal [11]. Also, a much simpler passive alternative consists in using an extra-cavity transparent material under conditions for nonlinear absorption so that the transmitted energy is stabilized [12]. Briefly, by integrating the Beer-Lambert law for multiphoton absorption as the laser beam of intensity $I_{0}$ propagates through a material of thickness $d$, the transmitted intensity I will decrease according to [13]: 
$(N-1) a_{N} d=\frac{1}{I^{N-1}}-\frac{1}{I_{0}^{N-1}}$,

where $a_{N}$ and $N$ are the multiphoton absorption coefficient and order, respectively. As for real laser sources, the energy is not stable and it fluctuates a certain quantity $\Delta I_{0}$. Applying error propagation theory to eq.(1) the fluctuation $\Delta I$ for the transmitted beam is:

$$
\frac{\Delta I}{I}=(1-A)^{N-1} \frac{\Delta I_{0}}{I_{0}},
$$

where $A$ is the obtained overall absorptivity along the crystal thickness. This results in the stabilization of the laser energy by the factor $(1-A)^{N-1}$, which scales with the material bandgap and irradiation wavelength (defining $N$ ) and the nonlinear absorption $(A)$ that can be handled by the material. Using this concept, several studies have focused on liquids such as benzene, dye-solutions, polymers, liquid crystals, inorganic composites, or nanoparticles $[12,14,15]$, and solids like perovskites [16], to prove laser stabilization in various regimes (from ns to fs). To our knowledge, such methods have not been used in the context of laser processing.

In this letter, we overcome the practical laser processing precision limits by a drastic reduction of pulse-to-pulse energy fluctuations by nonlinear absorption. We evaluate and compare the level of control on the laser fluctuations accessible under various configurations (intensity, materials, nonlinearities). Using the stabilized femtosecond beam for silicon amorphization, we quantify the enhancement of feature size reproducibility close to threshold. The results agree remarkably well with predictions from the noise model [7] and eq.(2). The validity of the approach is finally verified with a statistical demonstration using a large beam with peak fluences close to modification threshold revealing the possibility for significant performance improvement.

In the experiments, we use a laser amplifier (Pharos, Light Conversion) that delivers linearly polarized $<200 \mathrm{fs}$ pulses at a wavelength of $1030 \mathrm{~nm}$ and repetition rate of $20 \mathrm{kHz}$. The energy stability showed a standard deviation (SD, so-called RMS stability) of about $0.3 \%$. Given the nature of the experiment, the pulse-topulse fluctuation of our very stable system is at the sensitivity limit of the measuring devices (e.g. PE9-C, Ophir, noise of $0.04 \mu \mathrm{J}$ to be compared to measured energies of 10-60 $\mu$ ). To solve this technical issue, we artificially introduce larger fluctuations by controlling the laser attenuation (half-wave plate and polarizer at amplifier output). A central energy is set (0\%) and fluctuations are simulated by adjusting the transmittance $\pm 5 \%$ and $\pm 10 \%$ around this value, allowing accurate readings from a thermopile powermeter after integrating multiple pulses (Ophir 3A). The chosen fluctuations are quite large compared to most laser technologies available today. However, we expect that the gain expressed in eq.(2), and rigorously valid for small fluctuations, remains applicable for these conditions at which the energy stabilization and the produced features are readily measurable. To control the nonlinear absorption on the tested crystals, a telescope is built at the output of the laser and the crystal used for stabilization is placed in between the lenses (500 and $400 \mathrm{~mm}$ ) after the focal point (Supplement 1). The loose focusing conditions prevent propagation nonlinearities and beam distortions in air, an aspect confirmed by beam profile analyses without the crystal. In this manner by z-scanning the crystal along the optical axis we can change the interaction intensity and in turn the level of nonlinear absorption. We considered several materials (ZnSe, $\mathrm{Si}$, or sapphire). However, a compromise has to be found between high energy loss with low $N$ (e.g. ZnSe, Si) or working at conditions close to material damage for high $N$ (e.g. sapphire). Finally, we report our results on a ZnS crystal (3 mm thick, Edmund Optics) since, among the explored materials, its bandgap (3.9 eV) and properties gave the best compromise between energy stabilization and transmittance.

In Fig. 1 we plot the relative energy fluctuation versus the absorption of the beam. As the nonlinear absorption increases, the simulated energy fluctuations of \pm 5 and $\pm 10 \%$ are monotonously reduced to around $\pm 1 \%$ and $\pm 2 \%$, respectively. At around $A>0.4$ the stabilization saturates to a plateau, reaching a stabilization factor of around 1/5. Given the bandgap of $\mathrm{ZnS}$, the multiphoton order should be $N=4$ at the considered wavelength. This allows us to plot eq.(2) for the different incoming energy fluctuation levels (Fig. 1), finding a fairly good match with the experimental data for $A<0.4$. Despite the absorption keeps increasing, the apparent stabilization seems to saturate, which can be attributed to other loss processes such as inverse Bremsstrahlung absorption caused by electron avalanche. While these are also nonlinear processes from which we should expect a benefit for stabilization, the description would deviate from the simple power law expressed in eq.(2).
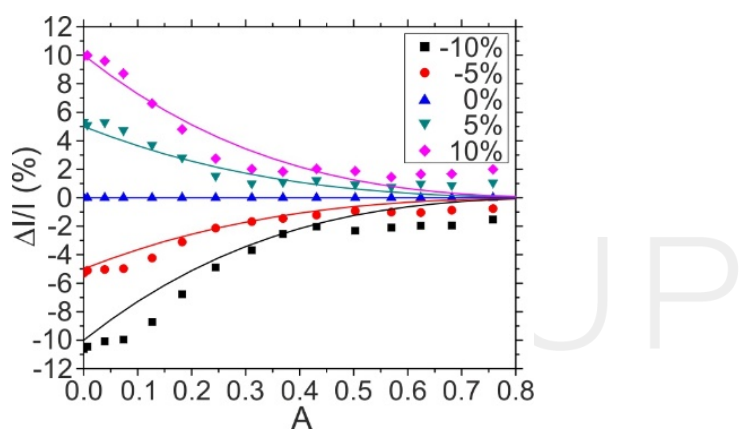

Fig. 1. Relative intensity fluctuations of $<200$ fs laser pulses at $1030 \mathrm{~nm}$ versus nonlinear absorption in the $\mathrm{ZnS}$ crystal. Fluctuations decrease with nonlinear absorption down to about $1 / 5$ of original fluctuations. The points correspond to measurements whereas the continuous lines are the fit of eq.(2) with the expected multiphoton order $\mathrm{N}=4$.

To explore how the energy stabilization can mitigate the produced features fluctuations, we irradiate a wafer of Si (111) relying on amorphization as it exhibits a threshold-based response even stricter than ablation [7]. In addition, the lower threshold for structural phase change from crystalline to amorphous allows us to work with larger beams [17]. Also, single pulse modification contours are easily measurable by optical means because of the higher reflectivity on the visible range of silicon in its amorphous phase and barely any change in topography [18]. During the irradiation experiments, the pumping energy on the crystal is not changed to keep a constant stabilization factor. Thus, we control the stabilized pulse energy reaching the sample by a half-wave plate and a polarizer after the telescope. Then, a 75-mm UV fused silica lens focuses the beam onto the sample, placed perpendicular to the beam on XYZ motorized stages. The precise positioning of the sample is monitored by an in situ microscope (10× objective, tilted at $45^{\circ}$ ) allowing to visualize its surface. The features are analyzed with an optical microscope (Nikon Lv-UEPI-N, $50 \times$ objective) which images are later systematically normalized and binarized to 
determine the amorphized surface and effective radii of the produced spots to avoid issues caused by different observer interpretations.

In Fig. 2a-b we represent the squared radius of the produced spots versus the pulse energy for $A=0.0$ and 0.3 . Both radii and energies are normalized to the beam waist $\omega$ and the threshold energy value $E_{t h}$ obtained by Liu's method without fluctuation ( $\left.0 \%\right)$ [19]. All data points show a monotonously increasing linear trend, indicating a rather good Gaussian energy distribution according to the considered energy range $I>I_{\max } / e$ above threshold. From the fits we obtain $\omega \approx 16 \mu \mathrm{m}$ in both cases but an $E_{\text {th }}$ that increases around four times for $A=0.3$ (Supplement 1). This leads to an inconsistent apparent threshold fluence, $F_{t h}=E_{t h} /\left(2 \pi \omega^{2}\right)$, which differs significantly from other reported values $[17,18,20]$. In this regard, we carefully analyzed the pulse width and beam spectrum, as well as its polarization, not finding any substantial change that can account for this inconsistency (Supplement 1). However, analyzing the spatial characteristics of the beam at the focal point, we identified a pedestal around the central Gaussian peak as the nonlinear absorption increases (Supplement 1). Despite this makes Liu's method not rigorously valid, it is worth noting the linear trend in Fig. 2a-b indicating that the top-part of the distorted profile remains relatively Gaussian and the pedestal barely contributes in the modification experiment. This is confirmed by accounting for effective energy values ignoring the pedestal energy (by numerical integration of measured profiles) [21]. Under this consideration, the two measurements conclude on the same threshold energy and fluence: $0.61 \pm 0.03 \mu \mathrm{J}$ and $0.16 \pm 0.02 \mathrm{~J} / \mathrm{cm}^{2}$, respectively. This is consistent with other values in the literature of $0.13-0.19 \mathrm{~J} / \mathrm{cm}^{2}$ $[17,18]$ and supported by theoretical models [20]. An important conclusion is the side effects of nonlinear absorption stabilization as the spatial beam profile can be potentially transformed. While it allows working at above threshold conditions in a certain range, it is a source of energy losses to be accounted in the analyses.
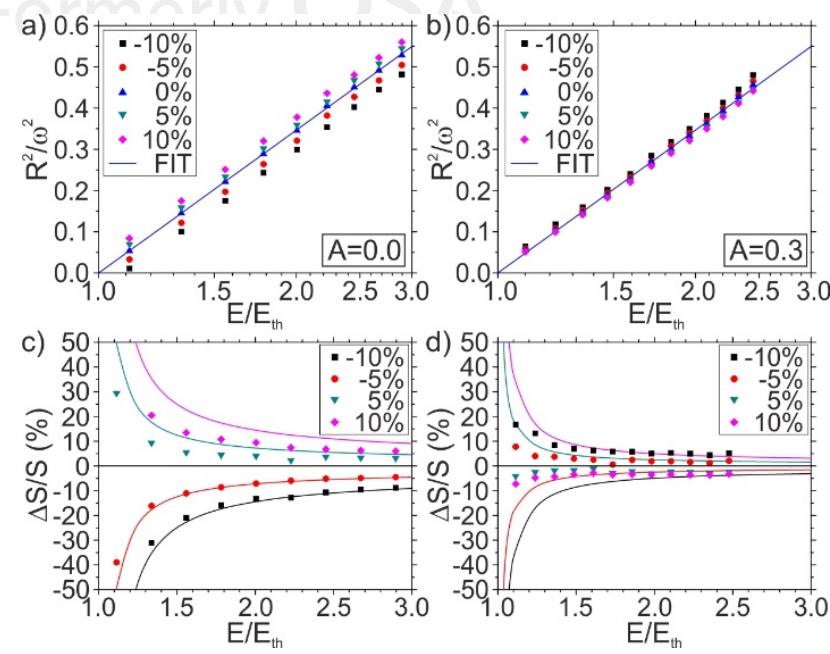

Fig. 2. Normalized squared radii versus pulse energy at simulated energy fluctuations of \pm 5 and $\pm 10 \%$ for a) $A=0.0$ and b) 0.3 . All points follow a linear trend, indicating a nearly Gaussian profile for the processing beam (continuous line). Normalized corresponding feature size fluctuations (area) versus pulse energy for c) $\mathrm{A}=0.0$ and $\mathrm{d}$ ) 0.3 . Continuous lines are the representation of eq.(3) with $\mathrm{N}=4$. Surface fluctuations increase as we approach the threshold. With nonlinear absorption the radii dispersion and surface fluctuations decrease. The noise model adequately fits the data.
When comparing the Liu's plot for $A=0.0$ and 0.3 in Fig. 2a-b, we can directly observe that as the nonlinear absorption increases there is less dispersion in the radii, which already indicates an improved reproducibility by laser stabilization. For quantitative analysis, in Fig. 2c-d we represent the surface variation $\Delta S$ as a function of the pulse energy $E$, respectively normalized to the average spot surface $S$ and $E_{t h}$. As expected, as we significantly exceed the threshold, the situation gets less sensitive to the laser fluctuations and a systematic reduction of the relative surface fluctuation is found. The introduction of nonlinear absorption is especially interesting in near-threshold conditions as it improves the reliability in high-resolution laser processing. Using our simple noise model under Gaussian beam approximation [7] we describe how the feature size fluctuation increases asymptotically as we approach the modification threshold. Accounting the multiphoton absorption laser energy stabilization stage, the model turns into:

$\frac{\Delta S}{S}=\left(\ln \frac{E}{E_{\text {th }}}\right)^{-1} \frac{\Delta E}{E_{0}}(1-A)^{N-1}$,

where $\Delta E / E_{0}$ corresponds to the relative energy variations at laser output set at $\pm 5 \%$ and $\pm 10 \%$ in our experiment. Taking $N=4$ (see above), the corresponding predictions are shown with continuous lines in Fig. 2c-d. The model reproduces well the experimental data with a progressive decrease of feature size fluctuations with the increase of absorption and/or set point energy. Negative energy fluctuations show a good match with the experimental data whereas better experimental improvements are found for positive ones. This dissymmetry might originate from relatively high fluctuation levels that deviate from adequate conditions to strictly apply the error propagation theory.

Another noticeable feature in Fig. 2b,d is a sign inversion of the surface variations with respect to the energy deviations when nonlinear absorption is introduced. This would mean that positive laser energy fluctuations result in smaller features than the original set point energy, and vice-versa. This is obviously unexpected but another consequence of the previously described change of the spatiotemporal characteristics of the beam that result in a threshold fluence variation depending on the energy reaching the crystal. While these aspects introduce complications in the descriptions and do not directly correspond to the multiphoton absorption only accounted in the model, it is interesting to note that it does not compromise the final objective of laser stabilization to improve the repeatability of the produced modifications. In addition, despite the limit of our model, it is observed in Fig. 2d that magnitudes of stabilization are relatively well described. Considering absolute values (ignoring the sign discrepancies) the measured performance is actually even better than the predicted one.

After these measurements and calculations supporting the concept, we finally carry out a practical demonstration of the applicability of the method. We perform matrices of irradiated spots at different energy levels relative to the threshold and compare the two stabilization cases. For this purpose, we induce artificial pulseto-pulse fluctuations that add to those of our stable laser source. After a calibration procedure, the rotation of the half-wave plate of the laser attenuator is computer-controlled so that the transmitted pulse energy follows a Gaussian white noise distribution with $\mathrm{SD}=2.2 \%$. For reliable statistics and a well-established distribution, we estimated the need for sample sizes $>50$ (Supplement 1 ).

Accordingly, we produced matrices of $64(8 \times 8)$ impacts at 
different energies relative to the threshold (Fig. 3). At first sight, we clearly observe that, as expected, spots are larger when the pulse energy is higher and the spot size distribution is more uniform with stabilization $(A=0.3)$. For conditions significantly exceeding the threshold $\left(1.05 E_{t h}\right)$, all impacts modify the sample because negative fluctuations are too small to lead to energy values below threshold. However, as we approach $E_{t h}$ the number of spots per matrix starts decreasing and the statistics of modification occurrence gives another way to reveal the stabilization (see also damage probability curves in Supplement 1). The position of the threshold is evident in the stabilized case since at $0.99 E_{\text {th }}$ we only observe one modification, which is not the case of the non-stabilized case where many random spots can still be found. Regarding the feature sizes, the detailed statistical analyses for the different matrix conditions are provided in Supplement 1 but it is interesting to note that for the stabilized case at $\approx 1.01 E_{t h}$, the radius of the 64 spots was $1.7 \pm 0.1 \mu \mathrm{m}$, which is the same average radius as for the non-stabilized case but with a larger distribution of $\pm 0.6 \mu \mathrm{m}$ (i.e. $35 \%$ fluctuations). While we did not targeted features as small as those obtained in super-resolution studies [4,5] and we intentionally used modest focusing conditions to simplify the conceptual demonstrations, it is also worth extrapolating the ability to achieve extremely small features. The excellent reproducibility obtained at $\approx 1.01 E_{\text {th }}$ for a resolution of $\approx \omega / 10$ with a relatively unstable source $(\mathrm{SD}=2.2 \%)$ holds promises for high-resolution demonstration with tightly focused pulses.

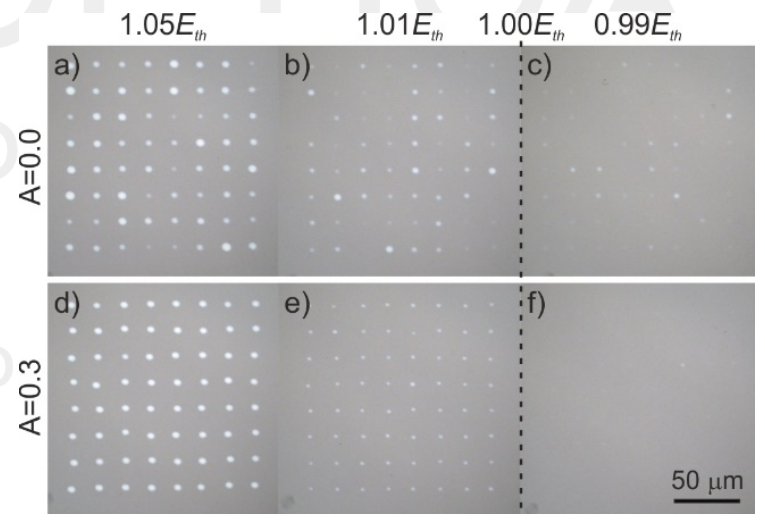

Fig. 3. Optical microscope images of femtosecond laser-induced amorphous spot matrices on $\mathrm{Si}$ (111) produced with original energy fluctuations of $\mathrm{SD}=2.2 \%$. a)-c) Without energy stabilization $(\mathrm{A}=0.0)$. d)-f) With extra-cavity energy stabilization $(A=0.3)$. Experiments are performed above $\left(1.05 E_{\text {th }}\right.$ and $\left.1.01 E_{\text {th }}\right)$ and below the fluence threshold $\left(0.99 E_{\text {th }}\right)$ clearly observing a reproducibility improvement when the beam is stabilized.

In this work, we proved the feasibility of using multiphoton absorption in a wide bandgap crystal to improve significantly the pulse-to-pulse energy stability of a femtosecond laser and enhance the precision limits of laser processing applications. While a degree of optimization surely remains by exploring different band gap material and wavelength combinations, we have concentrated on the reduction of energy fluctuations of an NIR-laser from an interaction in a $\mathrm{ZnS}$ crystal. The later permitted significant energy stabilization below damage threshold despite the observation of some nonlinear beam distortions in the experiments. By irradiating a Si (111) wafer, we observed the benefits of energy stabilization on the regularity of amorphous spots produced on its surface. Using a typical laser fluctuation of about $2 \%$, we unambiguously demonstrate a level of repeatability near threshold conditions that is unachievable otherwise. Interestingly, the approach allows reliable writing with a set point $<1.01 E_{t h}$. The corresponding feature size of $\approx \omega / 10$ observed in such conditions with large beams must directly translate in reliable writing capabilities with $10 \mathrm{~nm}$ resolution using tightly focused beams. Similar resolutions are already proved in laboratories [4,5] but the complexity causes precision to usually conflict with reliability. In that prospect, the implementation of our scheme holds potential to meet the mostdemanding precision manufacturing applications that are today addressed by extreme UV lithography and/or focused ion beam, and still remains a challenge for ultrafast laser writing.

Funding. H2020 European Research Council (No. 724480).

Disclosures. The authors declare no conflicts of interest.

Data availability. Data underlying the results presented in this Letter are not publicly available at this time but may be obtained from the authors upon reasonable request.

Supplemental document. See Supplement 1 for supporting content.

\section{References}

1. J. Cheng, C. Liu, S. Shang, D. Liu, W. Perrie, G. Dearden, and K. Watkins, Opt. Laser Technol. 46, 88-102 (2013)

2. F.J. Furch, W.D. Engel, T. Witting, A. Perez-Leija, M.J.J. Vrakking, and A. Mermillod-Blondin, Opt. Lett. 44, 4267-4270 (2019)

3. A.P. Joglekar, H. Liu, G.J. Spooner, E. Meyhöfer, G. Mourou, and A.J. Hunt, Appl. Phys. B Lasers Opt. 77, 25-30 (2003).

4. A.P. Joglekar, H.-H. Liu, E. Meyhofer, G. Mourou, and A.J. Hunt, Proc. Natl. Acad. Sci. 101, 5856 (2004)

5. Z. Li, L. Wang, H. Fan, Y. Yu, Q. Chen, and S. Juodkazis, Light Sci. Appl. 9, 41 (2020)

6. K. Sugioka, and Y. Cheng, Light Sci. Appl. 3, e149 (2014)

7. M. Garcia-Lechuga, G. Gebrayel El Reaidy, H. Ning, P. Delaporte, and D. Grojo, Appl. Phys. Lett. 117, 171604 (2020)

8. M. Garcia-Lechuga, O. Uteza, N. Sanner, and D. Grojo, Opt. Lett. 45, 952955 (2020)

9. P. Kühler, F.J. García de Abajo, J. Solis, M. Mosbacher, P. Leiderer, C.N. Afonso, and J. Siegel, small 5, 1825-1829 (2009)

10. M.S. White, R.W. Wyatt, and A.G. Brett, Opt. Commun. 44, 405-410 (1983)

11. S. Matsuo, L. Yan, J. Si, T. Tomita, and S. Hashimoto, Opt. Lett. 37, 16461648 (2012)

12. R.H. Pantell, and J. Warszawskim, Appl. Phys. Lett. 11, 213 (1967)

13. M. Grehn, T. Seuthe, M. Höfner, N. Griga, C. Theiss, A. MermillodBlondin, M. Eberstein, H. Eichler, and J. Bonse, Opt. Mater. Express 4, 689 (2014).

14. G.S. He, Progress in Optics 64, 155-278 (2019) ISSN 0079-6638

15. X. Feng, Z. Shi, J. Chen, T. Yu, X. Jiang, G. Du, J. Qiu, and S. Zhou, Adv. Opt. Mater. 8, 1902143 (2020)

16. T.C. Wei, S. Mokkapati, T.Y. Li, C.H. Lin, G.R. Lin, C. Jagadish, and J.H. He, Adv. Funct. Mater. 28, 1707175 (2018)

17. M. Garcia - Lechuga, N. Casquero, A. Wang, D. Grojo, and J. Siegel, Adv. Opt. Mater. 9, 2100400 (2021)

18. C. Florian, D. Fischer, K. Freiberg, M. Duwe, M. Sahre, S. Schneider, A. Hertwig, J. Krüger, M. Rettenmayr, U. Beck, A. Undisz, and J. Bonse, Materials 14, 1651 (2021)

19. J.M. Liu, Opt. Lett. 7, 196 (1982)

20. A. Ramer, O. Osmani, and B. Rethfeld, J. Appl. Phys. 116, 053508 (2014)

21. M. Garcia-Lechuga, and D. Grojo, Open Research Europe 1, 7 (2021) 


\section{References}

1. J. Cheng, C. Liu, S. Shang, D. Liu, W. Perrie, G. Dearden, and K. Watkins, "A review of ultrafast laser materials micromachining," Opt. Laser Technol. 46, 88-102 (2013)

2. F.J. Furch, W.D. Engel, T. Witting, A. Perez-Leija, M.J.J. Vrakking, and A. Mermillod-Blondin, "Single-step fabrication of surface waveguides in fused silica with few-cycle laser pulses," Opt. Lett. 44, 4267-4270 (2019)

3. A.P. Joglekar, H. Liu, G.J. Spooner, E. Meyhöfer, G. Mourou, and A.J. Hunt, "A study of the deterministic character of optical damage by femtosecond laser pulses and applications to nanomachining," Appl. Phys. B Lasers Opt. 77, 25-30 (2003).

4. A.P. Joglekar, H.-H. Liu, E. Meyhofer, G. Mourou, and A.J. Hunt, "Optics at critical intensity: Applications to nanomorphing," Proc. Natl. Acad. Sci. 101, 5856 (2004)

5. Z. Li, L. Wang, H. Fan, Y. Yu, Q. Chen, and S. Juodkazis, “O-FIB: far-fieldinduced near-field breakdown for direct nanowriting in an atmospheric environment," Light Sci. Appl. 9, 41 (2020)

6. K. Sugioka, and Y. Cheng, "Ultrafast lasers-reliable tools for advanced materials processing," Light Sci. Appl. 3, e149 (2014)

7. M. Garcia-Lechuga, G. Gebrayel El Reaidy, H. Ning, P. Delaporte, and D. Grojo, "Assessing the limits of determinism and precision in ultrafast laser ablation," Appl. Phys. Lett. 117, 171604 (2020)

8. M. Garcia-Lechuga, O. Uteza, N. Sanner, and D. Grojo, "Evidencing the nonlinearity independence of resolution in femtosecond laser ablation," Opt. Lett. 45, 952-955 (2020)

9. P. Kühler, F.J. García de Abajo, J. Solis, M. Mosbacher, P. Leiderer, C.N. Afonso, and J. Siegel, "Imprinting the optical near field of microstructures with nanometer resolution," small 5, 1825-1829 (2009)

10. M.S. White, R.W. Wyatt, and A.G. Brett, "A self-setting attenuator for laser pulse energy stabilization," Opt. Commun. 44, 405-410 (1983)

11. S. Matsuo, L. Yan, J. Si, T. Tomita, and S. Hashimoto, "Reduction of pulse-to-pulse fluctuation in laser pulse energy using the optical Kerr effect," Opt. Lett. 37, 1646-1648 (2012)

12. R.H. Pantell, and J. Warszawskim, "Laser power stabilization by means of nonlinear absorption," Appl. Phys. Lett. 11, 213 (1967)

13. M. Grehn, T. Seuthe, M. Höfner, N. Griga, C. Theiss, A. MermillodBlondin, M. Eberstein, H. Eichler, and J. Bonse, "Femtosecond-laser induced ablation of silicate glasses and the intrinsic dissociation energy," Opt. Mater. Express 4, 689 (2014).

14. G.S. He, "Multi-photon excitation based nonlinear optical effects and applications," Progress in Optics 64, 155-278 (2019) ISSN 0079-6638

15. X. Feng, Z. Shi, J. Chen, T. Yu, X. Jiang, G. Du, J. Qiu, and S. Zhou, "Allinorganic transparent composite materials for optical limiting," Adv. Opt. Mater. 8, 1902143 (2020)

16. T.C. Wei, S. Mokkapati, T.Y. Li, C.H. Lin, G.R. Lin, C. Jagadish, and J.H. He, "Nonlinear absorption applications of $\mathrm{CH} 3 \mathrm{NH} 3 \mathrm{PbBr} 3$ perovskite crystals," Adv. Funct. Mater. 28, 1707175 (2018)

17. M. Garcia - Lechuga, N. Casquero, A. Wang, D. Grojo, and J. Siegel, “ Deep silicon amorphization induced by femtosecond laser pulses up to the mid-infrared," Adv. Opt. Mater. 9, 2100400 (2021)

18. C. Florian, D. Fischer, K. Freiberg, M. Duwe, M. Sahre, S. Schneider, A. Hertwig, J. Krüger, M. Rettenmayr, U. Beck, A. Undisz, and J. Bonse, "Single femtosecond laser-pulse-induced superficial amorphization and re-crystallization of silicon," Materials 14, 1651 (2021)

19. J.M. Liu, "Simple technique for measurements of pulsed Gaussianbeam spot sizes," Opt. Lett. 7, 196 (1982)

20. A. Ramer, O. Osmani, and B. Rethfeld, "Laser damage in silicon: Energy absorption, relaxation, and transport," J. Appl. Phys. 116, 053508 (2014)

21. M. Garcia-Lechuga, and D. Grojo, "Simple and robust method for determination of laser fluence thresholds for material modifications: an extension of Liu's approach to imperfect beams," Open Research Europe 1, 7 (2021)

To cite this article:

Pol Sopeña, Mario Garcia-Lechuga, Andong Wang, and David Grojo,

"Ultrafast laser stabilization by nonlinear absorption for enhanced-precision material processing,"

Opt. Lett. 47, 993-996 (2022) 